\title{
Teorias e Protocolos para Simulações Quânticas de Dinâmicas Moleculares de Car-Parrinello e Metadinâmica
}

\author{
Flavio O. S. Neto, Hugo G. Machado, Eduardo C. Vaz, Yago S. \\ Silva \& Valter H. Carvalho-Silva
}

As simulações de dinâmicas moleculares, em síntese, fazem o uso de algoritmos adequados que tem por finalidade a resolução das equações de movimentos realizados pelos átomos e moléculas. Esta comunicação, trata-se de um guia básico a qual se pretende pontuar aspectos qualitativos sob uma visão generalizada, fornecendo um levantamento de suas aplicabilidades. Aqui, expomos o processo operacional, bem como conceitos específicos de metodologias derivadas, especificamente Dinâmica Molecular de Car-Parrinello e Metadinâmica. Os resultados apresentados têm função apenas de visualização para o leitor, fornecendo melhor entendimento.

Palavras chave: Dinâmica Molecular, DFT, CPMD, Metadinâmica.

The molecular dynamics simulations, in summary, make use of proper algorithms that have as ambit the resolution of the equations of motion performed by atoms and molecules. This communication is a basic guide, which aims to point qualitative aspects beneath a generalized view, providing a survey of its applicability. Here, we expose the practical operational process, as well as specific concepts of derived methodologies, specifically Car-Parrinello Molecular Dynamic and Metadynamics. The presented results have presented only for visualization, therefore it will make easier the reader have a better understanding.

Key words: Molecular Dynamic; DFT; CPMD; Metadynamic. 


\section{A Equação de Schrödinger}

A química quântica é o estudo de fenômenos químicos por meio da mecânica quântica, que tem como objetivo a resolução da Equação de Schrödinger (ES). A solução da ES trará conhecimentos acerca de propriedades atômicas e sistemas moleculares. ${ }^{1}$ A ES em sua forma mais simplificada, independente do tempo, está representada na Equação 1:

$$
H \psi(r)=\left[\frac{\hbar}{2 m} \nabla^{2}+V(r)\right] \psi(r)=E \psi(r)
$$

Tal equação possui solução analítica somente para sistemas muito simples. Sua resolução para sistemas mais complexos depende de aproximações. ${ }^{1}$

Os métodos aplicados na ES, tem por finalidade a busca por uma solução aproximada em um sistema multieletrônico. Destaca-se dentre estes, a Aproximação de Born-Oppenheimer (ABO), pois a mesma considera uma diferença significativa entre as massas dos núcleos e dos elétrons do sistema. Assumindo que os núcleos são estáticos, proporcionando a separação dos graus de liberdade eletrônicos e nucleares, permitindo efetuar a evolução dos núcleos sob a influência de um potencial. ${ }^{2}$

Esse potencial depende apenas de coordenadas eletrônicas, assim, os núcleos assumem coordenadas fixas e a ES é resolvida apenas para os elétrons na presença do potencial gerado pelos núcleos “estáticos”. Logo, a energia obtida, é a energia eletrônica, que é calculada através de outras aproximações, como é o caso de Hartree-Fock (HF), ou Teoria do Funcional da Densidade (no inglês, Density Functional Theory -DFT). ${ }^{3}$

\section{Dinâmica Molecular}

\section{DINÂMICA MOLECULAR CLÁSSICA}

A dinâmica molecular (DM) propõe a evolução temporal dos núcleos utilizando métodos de estrutura eletrônica (a exemplo de análogos de HF e DFT) viabilizando o estudo de: cinética de reações, visualização de vias e mecanismos específicos, processos de solvatação, como também podese obter diversas propriedades termodinâmicas e estudos para desenvolvimento de moléculas com propriedades farmacêuticas e/ou tecnológicas. ${ }^{1,4}$

$\mathrm{O}$ método de DM foi introduzido utilizando modelos de esferas rígidas, com choques elásticos para representar interações atômicas, o qual a dinâmica do sistema molecular é simulada pela integração numérica das equações de movimento. ${ }^{4,5}$

Tais equações de movimento são obtidas por meio das formulações clássicas de Hamilton:

$$
\dot{\boldsymbol{P}}_{I}=-\frac{\partial H}{\partial \boldsymbol{R}_{I}}
$$

e

$$
\dot{\boldsymbol{R}}_{I}=\frac{\partial H}{\partial \boldsymbol{P}_{\boldsymbol{I}}}
$$

no qual $R_{I}$ e $P_{I}$ são os parâmetros de posição e momento do do I-ésimo átomo do sistema, respectivamente. O Hamiltoniano clássico, chamado de $H$, pode ser definido como a soma da energia potencial e da energia cinética, como dado em:

$$
H\left(\boldsymbol{P}_{\boldsymbol{I}}, \boldsymbol{R}_{\boldsymbol{I}}\right)=\sum_{i=1}^{n} \frac{P_{I}^{2}}{2 M_{I}}+V\left(\boldsymbol{R}_{\boldsymbol{I}}\right)
$$

Portanto, pode-se obter as forças sobre os átomos por meio da derivada da energia potencial em relação à variação de posição atômica:

$$
F_{I}=M_{I} \ddot{\boldsymbol{R}}_{I}=-\nabla_{I} V\left(\boldsymbol{R}_{I}\right)=-\frac{d V}{d \boldsymbol{R}_{I}}
$$


Este método, entretanto, não era capaz de descrever os comportamentos eletrônicos do sistema uma vez que está alicerçado nos princípios de mecânica clássica, sendo assim, este primeiro método de dinâmica estudado foi chamado de dinâmica molecular clássica (DMC). ${ }^{5,6}$

\section{DINÂMICA MOLECULAR AB INITIO}

Quando é combinado o método de DMC juntamente a métodos destinados a descrever os comportamentos eletrônicos, é obtido um novo método chamado de dinâmica molecular ab initio (DMAI). Neste método uniu-se duas contribuições, onde os núcleos são tratados como partículas clássicas (contribuição obtida através da DMC) e os elétrons como partículas quânticas (contribuição obtida pelos métodos de estrutura eletrônica). ${ }^{5}$ Dentre alguns modelos de DMAI, podemos citar a dinâmica molecular de Ehrenfest (DME) ${ }^{7}$, dinâmica molecular de Born-Oppenheimer (DMBO) ${ }^{8,9}$ e a dinâmica molecular de Car-Parrinello (DMCP). ${ }^{10}$

Em princípio as equações de DMBO podem utilizar qualquer método de estrutura eletrônica. $\mathrm{Na}$ prática é necessário que se faça uma escolha criteriosa do método a ser usado levando em consideração o custo computacional agregado. A Teoria do Funcional de Densidade (DFT) é um dos métodos mais utilizados, sendo que este apresenta bom equilíbrio entre a exatidão dos cálculos e o custo computacional. ${ }^{9,11}$

Roberto Car e Michele Parrinello fizeram combinações das vantagens da DME e da DMBO e esta nova dinâmica foi então chamada de dinâmica molecular de Car-Parrinello em homenagem aos dois pesquisadores. ${ }^{5,12} \mathrm{Na}$ DMCP não é necessário a minimização da função de onda a cada passo de dinâmica realizado a exemplo da $\mathrm{DMBO}$, pois o subsistema eletrônico mantém-se minimizado durante a propagação dos núcleos. ${ }^{10}$

A ligação entre o tratamento recebido pelos elétrons e pelo núcleo é obtida por meio da lagrangiana estendida de Car-Parrinello ${ }^{10}$ representada na Equação 6:

$\mathcal{L}_{C P}=\frac{\mu}{2} \sum_{i=1}^{m}\left\langle\dot{\psi}_{i}(r) \mid \dot{\psi}_{i}(r)\right\rangle+\frac{1}{2} \sum_{I} \dot{M}_{I} \dot{R}_{I}^{2}-E_{K S}\left[\left\{\psi_{i}\right\},\left\{R_{I}\right\}\right]+\sum_{i, j=1}^{m} A_{i j}\left(\left\langle\psi_{i} \mid \psi_{j}\right\rangle-\delta_{i j}\right)$
Dentre as grandezas presentes na equação temos que, " $\mu$ " é um parâmetro de massa fictícia que controla a adiabaticidade. Este parâmetro impede as trocas de energia entre os subsistemas eletrônicos e iônicos. " $\mathrm{M}_{\mathrm{I}}$ " é a massa iônica real. "EKS" é o funcional energia de Kohn-Sham ${ }^{13}$ e " $A_{i j}$ " é um conjunto de multiplicadores de Lagrange que asseguram as restrições de ortonormalidade. ${ }^{1,5,10}$

As dinâmicas envolvendo trajetórias de BornOppenheimer e lagrangiana estendida assumem que uma simples superfície de potencial rege a dinâmica. Tais aproximações adiabáticas são amplamente utilizadas para análises de reações na superfície do estado fundamental. ${ }^{14}$ A maior limitação de dinâmicas adiabáticas são de que estas não são aplicáveis a reações envolvendo processos eletrônicos não-adiabáticos, como por exemplo cálculos de múltiplas superfícies de energia potencial. ${ }^{8,14}$

As duas aproximações mais amplamente utilizadas para considerar efeitos não-adiabáticos são a de surface-hopping e o método de Ehrenfest. ${ }^{7,14}$

Uma abordagem oriunda da DMCP que se mostra interessante no sentido de redução de tempo computacional é a Metadinâmica, principalmente para análise de reações químicas. A mesma é indicada para casos em que a caracterização do mecanismo reacional não é um problema, sendo já conhecido.

Essencialmente, o método consiste na adição de um termo extra à lagrangiana de CP. Este último, ficará encarregado em direcionar, de forma seletiva, o caminho à qual se elevará o progresso reacional em função do tempo. Transpondo matematicamente, tem-se:

$$
L_{M T D}=L_{C P}+\frac{1}{2} \sum_{\alpha} \mu s_{\alpha}^{2}-\frac{1}{2} \sum_{\alpha} k\left[s_{\alpha}(r)-s_{\alpha}\right]^{2}-V(t, s)
$$

sendo que $\mathrm{S}_{\alpha}$ (r) são as variáveis coletivas do sistema $(\mathrm{CV})$, as quais definem os estados das espécies moleculares (reagentes e produtos); $S$ e $\mu$, são respectivamente, as coordenadas e as massas das partículas fictícias que são acopladas às $\mathrm{CV}$ através de uma mola harmônica e por fim, $V(t, s)$, refere-se a um potencial externo dependente da história. ${ }^{15}$ 
Esse potencial pode ser descrito como uma somatória de gaussianas dispostas ao longo da trajetória do sistema, ao qual atua em um número selecionado de graus de liberdade. O mesmo fará uma "varredura" no espaço, expondo configurações de contorno já predispostas, aumentando a amostragem e reconstruindo a superfície de energia livre. ${ }^{16}$

Para compreender o efeito sobre a evolução do sistema, considera-se o caso simples do potencial unidimensional no gráfico abaixo.

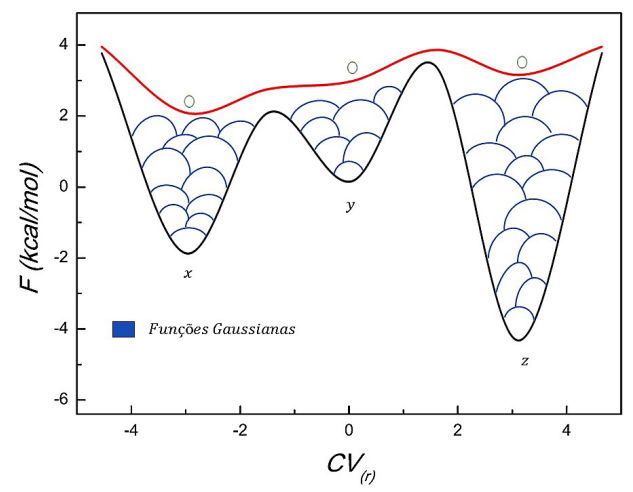

Figura 1. Propagação energética do sistema em função da variável coletiva.

Neste, os pontos $\mathrm{x}$, y e z representam os mínimos locais. Tomando como condição de início, um sistema localizado no ponto $\mathrm{y}$, submetido a uma simulação de dinâmica molecular convencional, estaria fadado ao confinamento em dada região, sendo necessário um tempo de simulação muito grande para observar o processo reativo. Tal situação se sustenta em função da barreira energética ser maior que a liberação de energia do sistema. Assim, conforme a deposição das funções gaussianas, levantavase os potenciais de polarização do sistema, repaginando a paisagem de energia livre. Difundindo-se ao longo dos demais mínimos, a ascensão promovida resultará na evolução reacional do sistema em um tempo inferior aqueles fornecidos por dinâmicas convencionais. ${ }^{17}$

Exemplos de variáveis coletivas que se faz menção, incluem, comprimento de ligação, ângulos de ligação e diedrais, números de coordenação entre outros, ficando o critério de escolha dependente da reação de interesse. Além dessas variáveis mencionadas, a altura e propagação das gaussianas, a massa da partícula fictícia e a constante de mola $(\mathrm{k})$, constituem parâmetros que controlam o caráter das dinâmicas, dos quais precisam ser ajustados para otimizar a acurácia dos cálculos. ${ }^{15}$

Além de mapear de forma mais intuitiva as etapas reacionais, a Metadinâmica toma como vantagem a não necessidade de uma estimativa energética inicial para sua extrapolação, com adendo do controle térmico constante durante a simulação e também da possibilidade de contabilizar os efeitos de vizinhança. ${ }^{17}$

\section{DINÂMICA MOLECULAR QUÂNTICA}

Por outro lado, existe um método a qual aplica-se correções quânticas para os graus de liberdades nucleares, aos quais são inseridos em simulações de dinâmicas moleculares baseado na expressão de caminho integral de Feynman.

Matematicamente, no caso clássico, as partículas se movimentam apenas em uma dimensão espacial, ou seja, existe apenas uma trajetória a qual interliga pontos no espaço-tempo através das equações clássicas 2 e 3 . Sob outra perspectiva, na mecânica quântica, outras trajetórias são permitidas, onde o deslocamento de uma coordenada inicial à final é quantificado probabilisticamente, seguindo uma distribuição de trajetórias. ${ }^{18}$

Neste, também se faz uso da aproximação de BornOppenheimer, entretanto, as posições nucleares podem ser "fragmentadas" em partículas fictícias, comumente chamadas de beads, as quais estão conectadas por potenciais harmônicos regidos por um hamiltoniano efetivo. Diante disso, o átomo, antes tratado como uma entidade localizada, aumenta seu caráter ondulatório, passando a ser um ente deslocalizado, potencialmente susceptível a efeitos quânticos relevantes, tal como o tunelamento e energia de ponto zero. ${ }^{19}$ Entretanto, a discussão desse tópico está além do escopo desse trabalho e será discutido em trabalhos futuros. 


\section{DINÂMICA MOLECULAR COM SOLVENTES}

\section{Função de Distribuição Radial de Pares - $g(r)$}

Durante a dinâmica molecular com solventes é preciso analisar o ordenamento do sistema. Uma vez que a descrição de um sistema em fase líquida é possível somente em termos probabilísticos, devido ao fato de um líquido consistir de um arranjo desordenado de átomos ou moléculas. Esta análise pode ser realizada por meio do cálculo da função de distribuição radial de pares (FDRP). ${ }^{5}$

A função pode ser descrita como a correlação de partículas em um sistema molecular de muitas moléculas. ${ }^{20}$ Para que possamos calcular, selecionamos uma partícula como origem e temos que o número médio de partículas a uma distância r e r+dr é igual a $\rho g(r) d r$, sendo que g(r) é a função de distribuição radial de pares. ${ }^{20,21}$

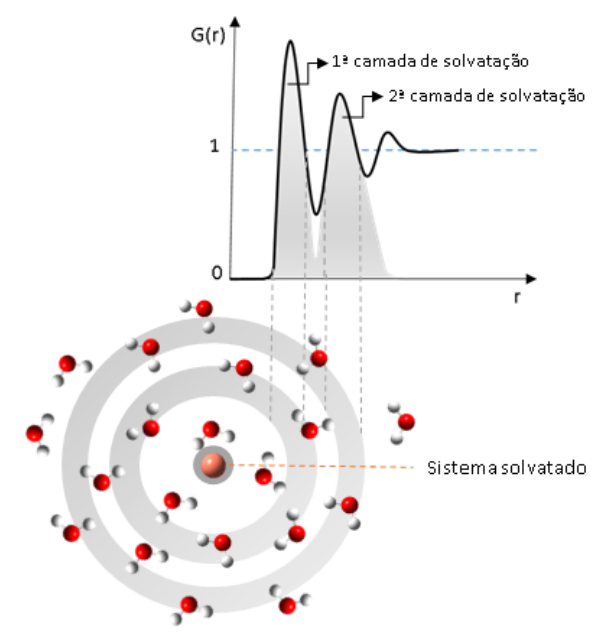

Figura 2. Figura esquemática de $\mathrm{g}(\mathrm{r})$ representando os picos da FDRP e a distribuição das moléculas do líquido analisado.

Adotando-se $\mathrm{N}$ como número de partículas encontradas em uma camada esférica de raio $r$, e tendo o termo de volume como $4 \pi r^{2} \Delta r$, podemos encontrar a equação da FDRP:

$$
N(r, r+\Delta r)=\rho g(r) 4 \pi r^{2} \Delta r
$$

Isolando $\mathrm{g}(\mathrm{r})$, temos:

$$
g(r)=\frac{N(r, r+\Delta r)}{4 \pi r^{2} \Delta r \rho}
$$

sendo que $\mathrm{N}(\mathrm{r}, \mathrm{r}+\Delta \mathrm{r})$ é o número médio de átomos encontrados na camada esférica e $\rho$ é a densidade média de átomos na solução. ${ }^{20}$

\section{Tempo Médio de Residência}

O tempo médio que uma partícula se mantem em uma determinada região monitorada do espaço é chamado de Tempo Médio de Residência (TMR). Essa análise de tempo em que a molécula reside em determinada região é fundamental para estudos de difusão de átomos e moléculas em uma solução. ${ }^{21,22}$

Em uma análise de TMR, o tempo de residência se inicia a partir da entrada de uma partícula na primeira camada de solvatação, sendo que essa camada é delimitada pelos valores obtidos por meio dos cálculos de FDRP. ${ }^{21}$

Após quantificar e expor os principais métodos de dinâmica molecular e alguns de seus fundamentos, este artigo propõe guiar o leitor através de protocolos práticos para aplicação dessas metodologias teóricas, gerando arquivos de entrada (input) e saída (output) através de pacotes computacionais.

\section{Metodologia e Procedimentos}

Primeiramente, é necessário destacar que a metodologia e os procedimentos que serão apresentados neste capítulo são exclusivamente baseados em processos para dinâmicas calculadas com o uso do software CPMD. ${ }^{23}$ Em segundo lugar, vale destacar que não focaremos no procedimento para a DME.

Sendo assim, para as simulações computacionais, recomenda-se a construção estrutural do sistema sob investigação, buscando a proximidade de estruturas geométricas e estados de energia estáveis em função aos referenciais experimentais. Esse tipo de minimização é uma pré-otimização, a qual fornece resultados sensivelmente 
próximos da disposição de equilíbrio de energia, diminuindo os custos computacionais subsequentes. Este processo pode ser viabilizado através do pacote computacional packmol. ${ }^{24,25}$

Após a minimização é recomendado salvar o arquivo em formato .xyz no qual está presente as coordenadas cartesianas do sistema. Este formato é necessário para a construção do input para o CPMD. Há alguns softwares que geram o arquivo .xyz com o número atômico indicando um átomo. Nesses casos é necessário substituir o número atômico pelo símbolo do átomo e também indicar na primeira linha do arquivo a quantidade de átomos do sistema, assim como na figura abaixo:

\begin{tabular}{|c|c|c|c|}
\hline \multicolumn{4}{|c|}{712} \\
\hline C & 16.386524 & 3.520549 & 2.255929 \\
\hline $\mathrm{H}$ & 15.990034 & 4.155192 & 2.858474 \\
\hline $\mathrm{H}$ & 16.551390 & 3.942789 & 1.410759 \\
\hline $\mathrm{H}$ & 17.214753 & 3.204510 & 2.623883 \\
\hline C & 13.626248 & 3.661638 & 0.515779 \\
\hline C & 14.398330 & 3.651377 & -0.623433 \\
\hline $\mathrm{H}$ & 15.106434 & 3.052647 & -0.695739 \\
\hline C & 14.129116 & 4.526640 & -1.666238 \\
\hline $\mathrm{H}$ & 14.648699 & 4.521510 & -2.437495 \\
\hline C & 13.085685 & 5.394721 & -1.534481 \\
\hline C & 12.264314 & 5.423452 & -0.424192 \\
\hline $\mathrm{H}$ & 11.550413 & 6.017050 & -0.366347 \\
\hline C & 12.543612 & 4.539979 & 0.594511 \\
\hline C & 10.693552 & 5.232597 & 1.960280 \\
\hline C & 9.528407 & 4.897575 & 1.323992 \\
\hline $\mathrm{H}$ & 9.503445 & 4.187514 & 0.723054 \\
\hline c & 8.395252 & 5.630724 & 1.590719 \\
\hline $\mathrm{H}$ & 7.595332 & 5.420886 & 1.164920 \\
\hline C & 8.442044 & 6.661954 & 2.477665 \\
\hline $\mathrm{H}$ & 7.676912 & 7.160126 & 2.651198 \\
\hline C & 9.613110 & 6.964654 & 3.109132 \\
\hline $\mathrm{H}$ & 9.637200 & 7.669584 & 3.714891 \\
\hline c & 10.747287 & 6.251001 & 2.866508 \\
\hline $\mathrm{H}$ & 11.540215 & 6.453143 & 3.306767 \\
\hline $\mathrm{N}$ & 13.849891 & 2.835627 & 1.635066 \\
\hline $\mathrm{H}$ & 13.361869 & 3.050595 & 2.440709 \\
\hline $\mathrm{N}$ & 12.812955 & 6.354124 & -2.606208 \\
\hline 0 & 15.765691 & 1.406783 & 0.917797 \\
\hline 0 & 15.070668 & 1.523759 & 3.286361 \\
\hline 0 & 13.615160 & 6.444421 & -3.521594 \\
\hline 0 & 11.820587 & 7.054951 & -2.529082 \\
\hline 0 & 11.848678 & 4.462509 & 1.780802 \\
\hline a & 15297545 & 2173844 & $=\pi 32264$ \\
\hline
\end{tabular}

Figura 3. Exemplo de arquivo .xyz corrigido com os símbolos dos átomos e com a quantidade de átomos.
Após formatação do arquivo .xyz é usado um software, desenvolvido pelo nosso grupo de pesquisa para criar os inputs automaticamente, chamado de gqtea ${ }^{\circledR}$. O software tem diversas outras funções além da mencionada, sendo não adequado o aprofundamento neste artigo. A utilização do gqtea não inviabiliza o processo de construção dos inputs, mas facilita significativamente. O software foi escrito em linguagem Fortran, basta ser executado e selecionado a opção correspondente à criação de input utilizando um arquivo .xyz, como demonstrado na Figura 4 e os inputs serão gerados:

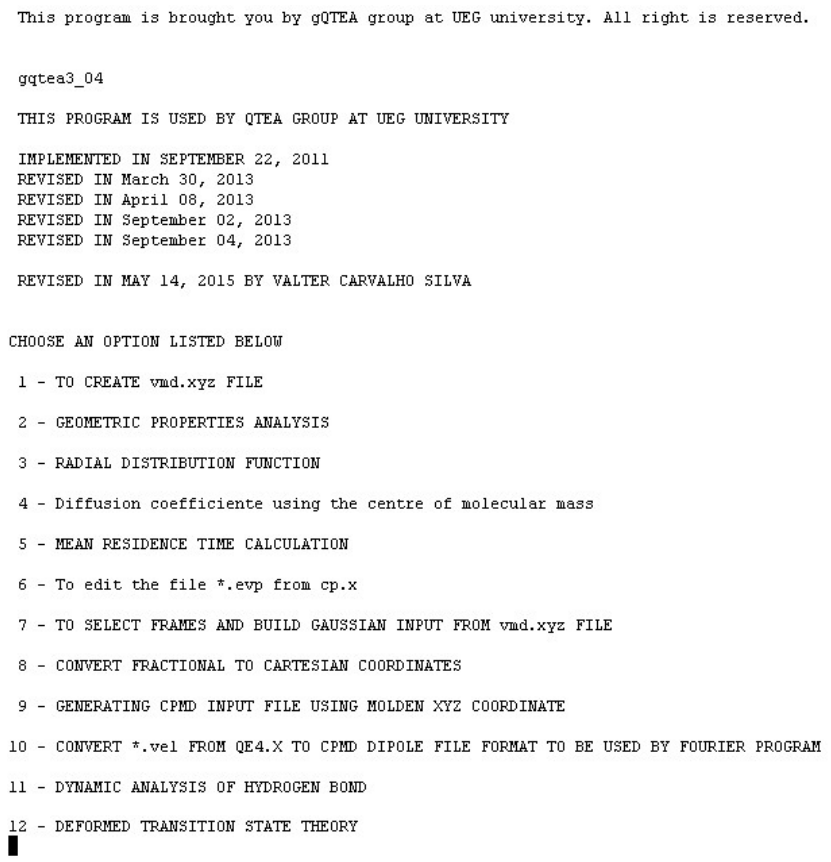

Figura 4. Interface do gqtea ${ }^{\circledR}$.

Como pode ser observado, deve-se utilizar a opção “9”, neste caso, para gerar os inputs. Será necessário fornecer o nome do arquivo com formato .xyz para que o software possa ler. 


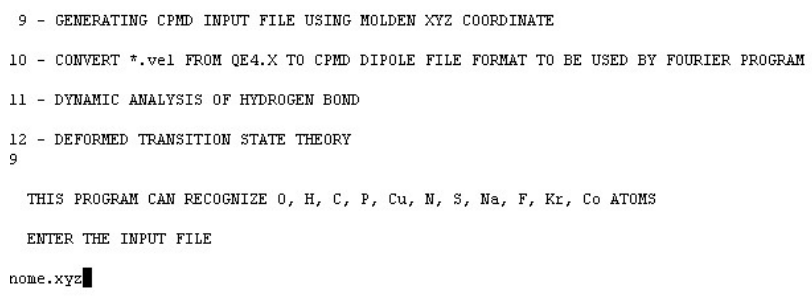

Figura 5. Opção 9 do software solicita ao leitor o nome do arquivo com formato .xyz.

Dois arquivos de inputs serão gerados após o termino do processo do software. O primeiro input (cpmd_simulation. inp) é referente à simulação de dinâmica molecular que será realizada. O segundo (cpmd_wfnop.inp) se trata do input de minimização de função de onda, o qual deve ser rodado primeiro.

Após a criação dos inputs o leitor deverá decidir por alguns pontos básicos, entretanto de grande importância:

- Qual método utilizar? (

- Qual número de passos de dinâmica quero simular? (2)

- Qual temperatura em que acontecerá a dinâmica? (3)

- Qual funcional DFT utilizar? (4)

- Qual carga e simetria do sistema? (5)

- Qual tamanho do sistema em angstroms? (6)

- Qual pseudopotencial utilizar? (7)

Os números que foram destacados devem ser analisados pelo leitor para melhor atender sua necessidade de cálculo. Tais pontos são indispensáveis de serem analisados antes de submeter o input para ser calculado.

O último passo a ser destacado antes de submeter o input para o cálculo, é obter os pseudopotenciais referentes aos átomos e ao funcional escolhido para a dinâmica. Sem a presença destes arquivos no mesmo diretório em que o input está presente, o cálculo não irá acontecer. Caso o leitor desejar deixar os arquivos de pseudopotenciais em outro diretório, o mesmo deve ser especificado na linha de comando de submissão do cálculo por meio do argumento PP_path.

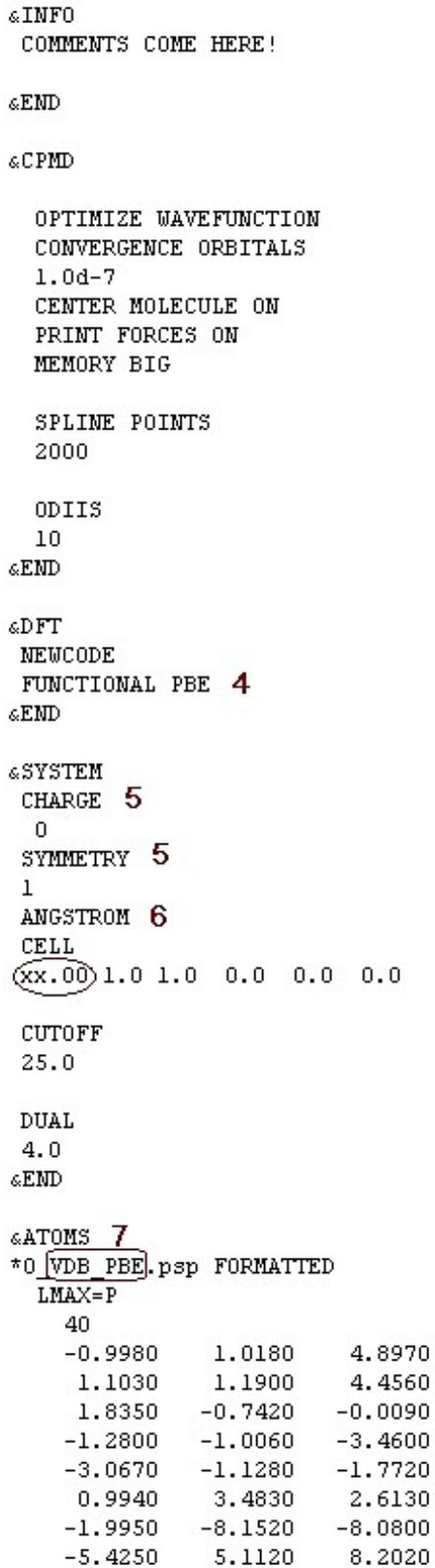

Figura 6. Modelo do input cpmd_wfnop.inp com as numerações referentes destacadas no texto. 


\section{Artigo Convidado 1}

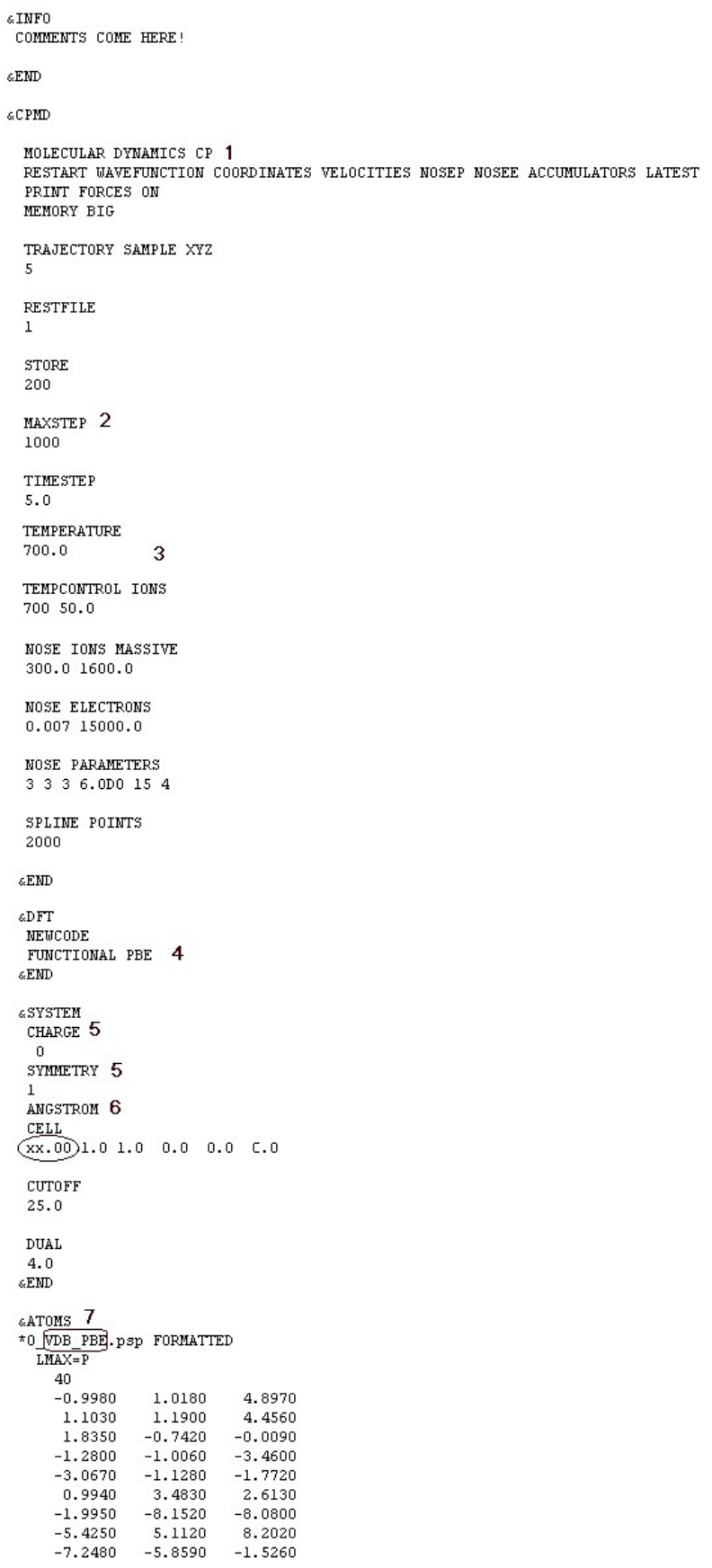

Figura 7. Modelo do input cpmd simulation.inp com as numerações referentes destacadas.
Após toda organização do diretório e do input a submissão do cálculo é feita no pacote computacional CPMD. Sendo que, novamente, primeiro deve-se minimizar a função de onda e, somente após, deve-se submeter o arquivo de simulação.

Vale ressaltar que este artigo traz as informações de modo introdutório, sendo que, se necessário, o leitor deve consultar o manual online disponibilizado pelo CPMD ${ }^{23}$ para obtenção de informações mais aprofundadas.

\section{Resultados e Discussões}

Para confirmar o término dos cálculos, o leitor pode visualizar o output do gerado pelo CPMD, assim como demonstradas na Figura 8.

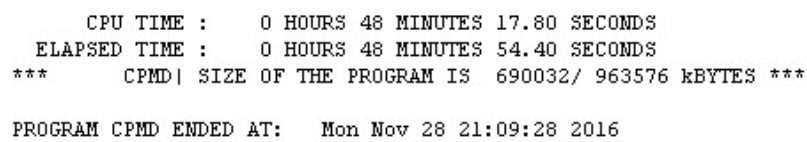

Figura 8. Informações sobre o cálculo emitidas nos outputs.

Adicionalmente, o leitor poderá encontrar em seu diretório alguns arquivos provenientes das simulações de dinâmica, sendo alguns deles: ENERGIES, GEOMETRY, GEOMETRY.xyz, LATEST, RESTART.1, TRAJECTORY, TRAJEC.xyz.

$\mathrm{O}$ arquivo ENERGIES contém todos os valores de energia durante toda a trajetória de simulação de dinâmica. Dentre os valores estão presentes a energia cinética fictícia dos elétrons em unidade atômica, a energia de KohnSham (que equivale à energia potencial de uma dinâmica clássica), energia clássica (correspondente à soma da energia de Kohn-Sham com energia cinética dos íons), e o Hamiltoniano de energia (correspondendo à soma dos valores de energia clássica com energia cinética dos elétrons. $\mathrm{O}$ arquivo sai pronto para uso com os respectivos valores e poderão ser analisados em softwares de análise gráfica, assim como demonstrado abaixo: 


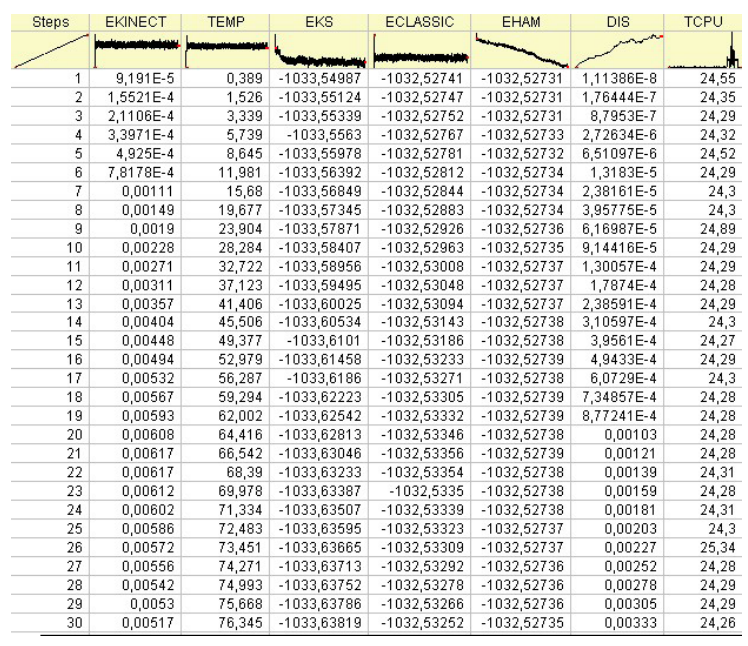

Figura 9. Arquivo ENERGIES, seus valores e curvas de energia.

Um ponto muito importante a ser considerado nas análises de energia da dinâmica é a adiabaticidade do sistema garantindo que a dinâmica está sendo simulada em uma superfície de Born-Oppenheimer. Deve-se verificar a troca entre as energias eletrônicas e nucleares do sistema. Caso haja a troca (por meio da análise do cruzamento das curvas), a dinâmica é inválida e deve-se tentar melhorar os parâmetros de minimização da função de onda para desfavorecer a troca destas energias.

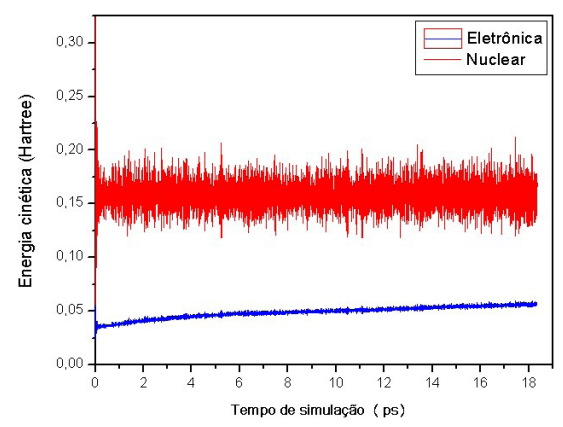

Figura 10. Representação das energias eletrônicas e nucleares do sistema, de acordo com a ABO. Para que possa ser analisado a adiabaticidade da dinâmica deve-se correlacionar a energia cinética eletrônica com a energia cinética iônica. Para tal correlação é necessário subtrair os valores da energia clássica dos valores da energia de Khon-Sham (ECLASSIC-EKS) obtendo a componente nuclear/iônica Após a obtenção dos valores, basta plotar juntamente o valor da energia cinética eletrônica (EKINECT).
$\mathrm{O}$ arquivo GEOMETRY contém as atuais posições e velocidades iônicas da simulação em relação aos passos da dinâmica em unidade atômica. Enquanto que o arquivo GEOMETRY.xyz contém as mesmas informações, porém com unidade em angstroms.

$\mathrm{O}$ arquivo LATEST contém as informações do último arquivo de restart da dinâmica. O arquivo RESTART.1 contém os velhos e os novos arquivos para recomeçar o cálculo da dinâmica.

O arquivo TRAJECTORY contém todas as velocidades e posições iônicas durante toda a trajetória da dinâmica. Enquanto que o arquivo TRAJEC.xyz contém as mesmas informações, porém no formato .xyz, o qual é compatível para visualização e análise da dinâmica.

Com o auxílio de um software de visualização, é possível visualizar a dinâmica e também obter informações da mesma, tais como comprimento de ligação entre dois átomos do sistema, valores de angulação referentes à três átomos do sistema, ângulos diedrais, assim como também superfícies de potencial eletrostático, gráficos das informações citadas, orbitais dos átomos do sistema, dentre outras funções.

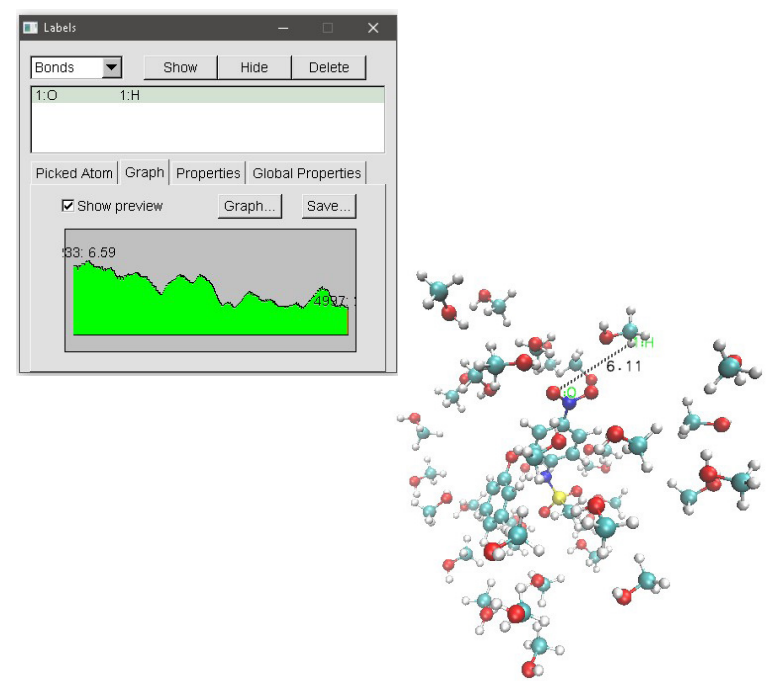

Figura 11. Representação do passo inicial da dinâmica com avaliação e caracterização gráfica de comprimento de ligação de dois átomos. ${ }^{26}$ 
O software de visualização utilizado pelo nosso grupo de pesquisa oferece opções de exportação do gráfico de comprimento de ligação, ângulos de ligação, dentre outras propriedades geométricas, para análises mais profundas em softwares gráficos especializados.

Os resultados de dinâmica molecular sempre são tratados conjuntamente com os resultados de cálculos estáticos e ou análises de estruturas cristalográficas. Por meio de análises de propriedades geométricas é possível validar os cálculos estáticos e a dinâmica molecular comparados à(s) estrutura(s) cristalográfica(s).

Caso a dinâmica seja realizada em solução, é possível realizar algumas análises por meio dos resultados dos cálculos de dinâmica. Tais análises comprovam ou reprovam a solubilidade de uma determinada molécula em um determinado meio.

Por meio do gqtea é possível fazer as análises de FDRP e TMR, opções "3" e "5" respectivamente do software.

Os gráficos de FDRP irão delimitar as chamadas "camadas de solvatação" ou "shells de solvatação". Tais camadas representam uma região em que consideramos perto o bastante para solvatar a molécula sob análise.

Vale ressaltar que os valores de FDRP considerados válidos para análise são superiores a 1 , uma vez que por se tratar de uma função de média, deve-se observar apenas as interações acima da média da função.

A figura abaixo representa uma análise de FDRP.

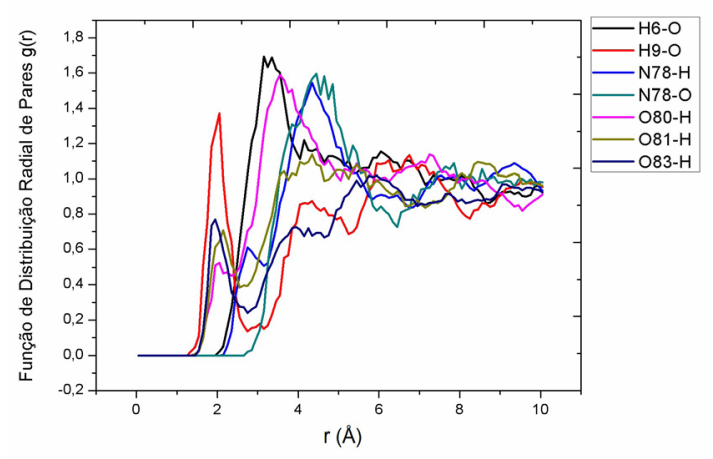

Figura 12: Representação gráfica de uma análise da FDRP.
Uma vez delimitado as camadas de solvatação das interações de interesse, parte-se para a análise de TMR, onde verifica-se por qual quantidade de tempo a molécula de solvente permanece dentro desta camada.

Se houver a presença de uma ou mais moléculas de solvente dentro da camada de solvatação por um longo período de tempo, é considerado como "solúvel" a molécula sob análise. Essa solubilidade pode ser alta ou baixa, dependendo de análises mais específicas para quantificar.

A figura abaixo apresenta a presença de várias moléculas de solvente dentro da primeira camada de solvatação durante todo o cálculo de dinâmica.

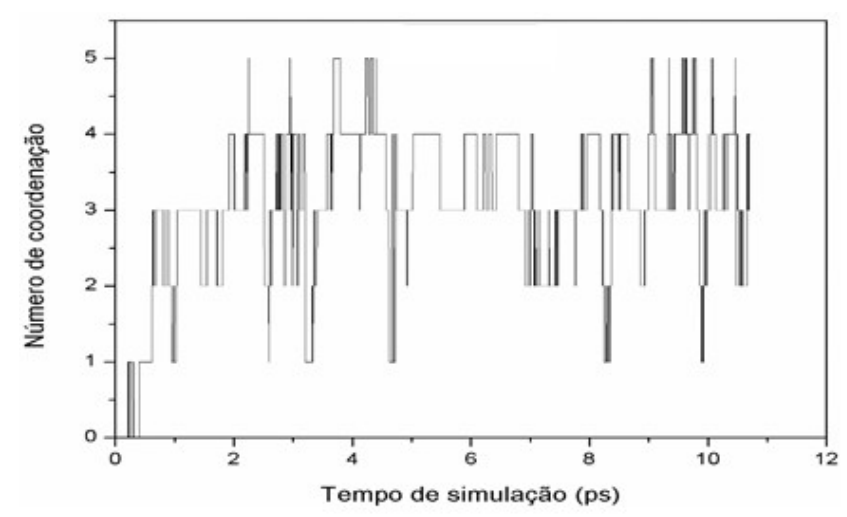

Figura 13: Representação gráfica de uma análise de TMR.

Após análises teóricas é recomendado a corroboração com resultados experimentais e vice-versa, como já mencionado previamente. Sendo assim, citando e exemplificando algumas análises - de várias possíveis a serem realizadas com dinâmica molecular - é possível verificar a importância de tal método e justificar o aumento exponencial de seu uso em trabalhos atuais.

\section{METADINÂMICA}

Para sistemas reacionais onde a barreira energética é 
muita alta, o processo de dinâmica convencional necessita de muito tempo para contabilizar uma reação, processo conhecido como eventos raros. Para contornar este gargalo, logo abaixo é apresentada as etapas de cálculo da metadinâmica que força a ocorrência da reação química. O processo de submissão dos cálculos em metadinâmica se apresenta de forma análoga as etapas anteriormente descritas em CPMD. Vale a ressalva, em casos cujo o sistema exiba multiplicidade diferente do estado singleto, a necessidade do incremento da variável LSD (Local SpinDensity), que atuará no auxílio da minimização da função de onda.
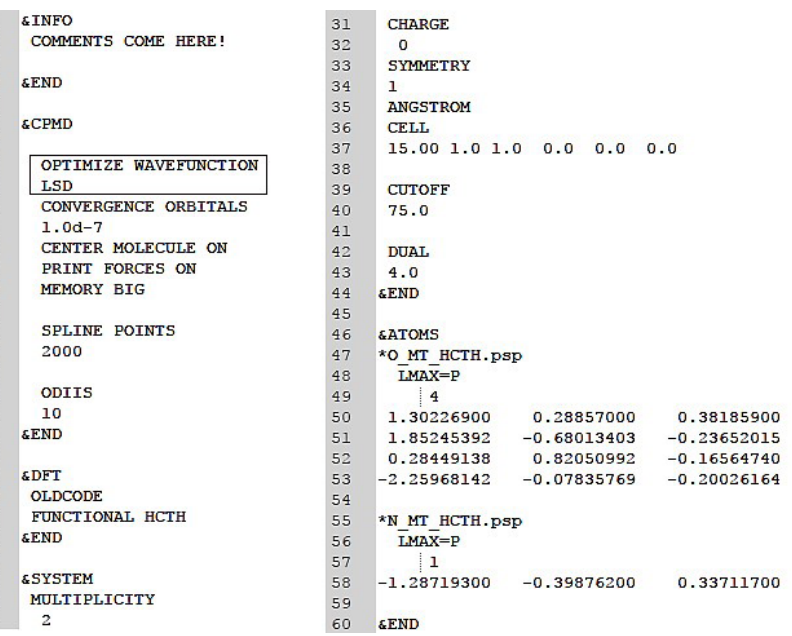

Figura 1: Modelo do input mtd_wfnop.inp para reação atmosférica $\mathrm{NO}+\mathrm{O}_{3}$.

Já em função do arquivo de simulação propriamente dito, deve-se notar as definições de coordenadas reacionais dos átomos, uma vez que parâmetros específicos que facilitam o processo de convergência reacional devem ser implementados. Aqui, não será discutido detalhadamente todos os parâmetros para rodar metadinâmica focando somente naqueles que são indispensáveis para a simulação. Maiores detalhes podem ser encontrados no manual online do CPMD.

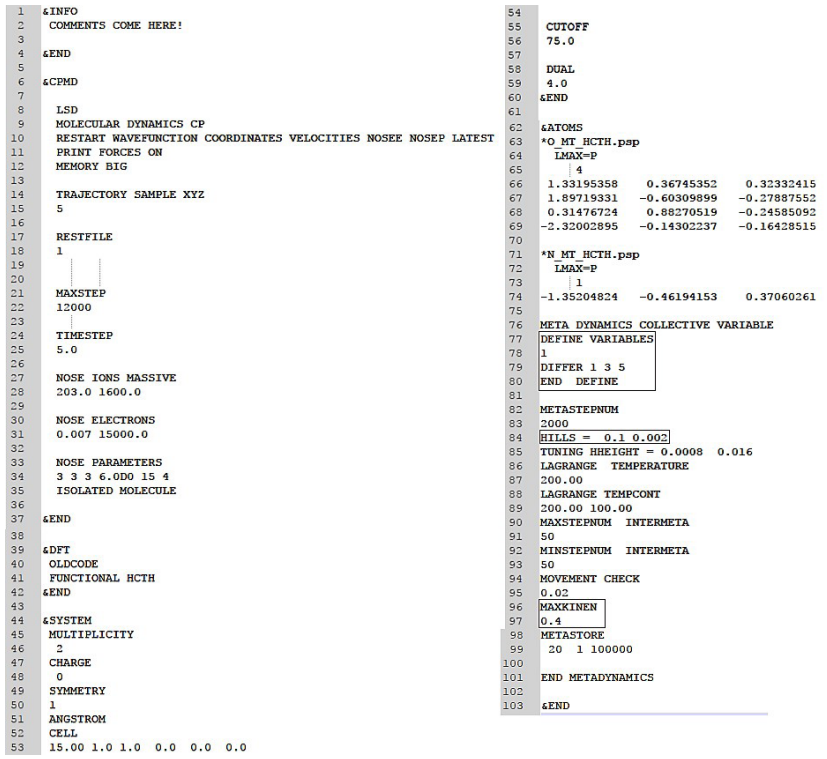

Figura 2: Modelo do input mtd_simulation.inp para a evolução temporal da reação $\mathrm{NO}+\mathrm{O}_{3}$.

Destacamos três principais variáveis que atuarão de forma enfática no progresso reacional. A primeira está relacionada com as variáveis coletivas (quantitativamente e qualitativamente). As coordenadas de reações podem ser implementas em função de várias coordenadas ou somente uma. A váriavel Differ, empregada no exemplo exposto para reação do $\mathrm{O}_{3}$ com $\mathrm{NO}$, será o primeiro caso analisado.

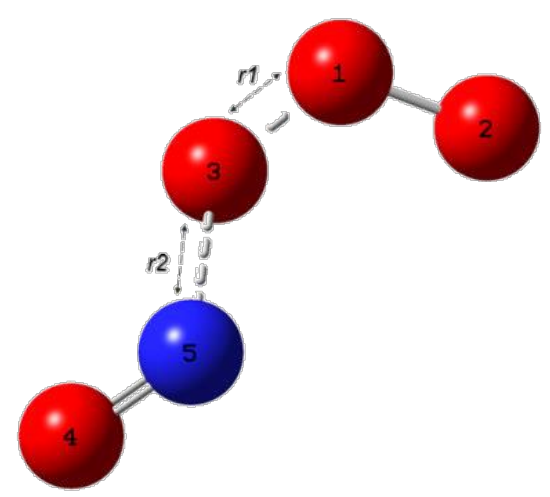

Figura 3: Estrutura das espécies reagentes com numeração atômica adotada nos cálculos. 
Considerando a transferência do átomo de oxigênio (Figura 3), a escolha por uma variável definida como coordenada de reação $(\Delta \mathrm{r})$, sendo a diferença da quebra da ligação, r2, dado pela ligação N5---O3, e a formação da ligação, r1, dado pela ligação O3---O1, comumente consegue descrever a etapa reativa. Quando $\Delta \mathrm{r}<0$, representa a condição dada por reagentes e $\Delta r>0$, evidencia a formação do produto.

Considerando o input de cálculo do CPMD, o parâmetro "Hills" está relacionado ao formato das funções gaussianas que serão acopladas ao sistema. O primeiro termo define a largura da função, e o segundo está vinculado à altura da mesma.

O último indicador em destaque é o "Maxkinen", ao qual representa a energia cinética máxima que os elétrons podem adquirir para que não ocorra uma violação na superfície de Born-Oppenheimer no estado fundamental.

Após a conclusão das etapas descritas genericamente, há uma necessidade de tratamento mediante ao código Vreco. inp disponível no pacote CPMD, mediando o levantamento de gráficos e superfícies energéticas.

Tomando como referência a reação exemplificada anteriormente, pode-se fazer a análise da energia livre de Helmholtz (F) em função da coordenada de reação $(\Delta \mathrm{r})$.

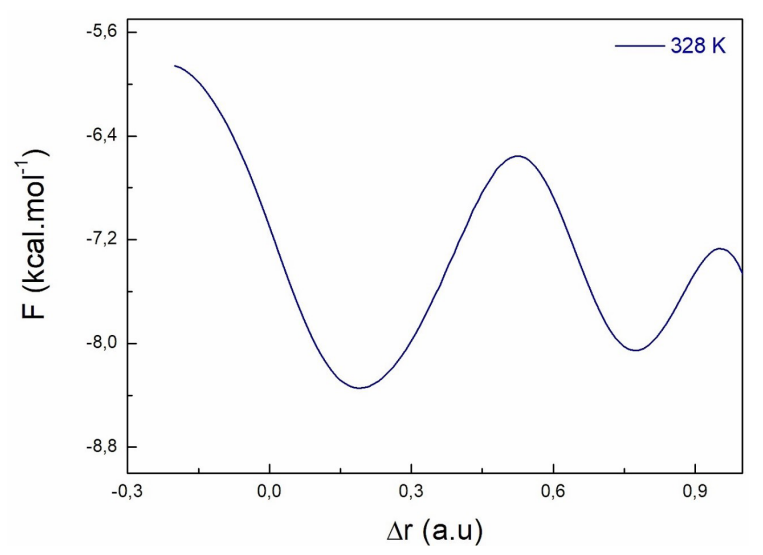

Figura 4: Curvas de energia livre para o processo de degradação do ozônio.
Por definição, e adotando aproximações, mediante ao perfil apresentado, pode-se pontuar a região do complexo ativado $(\Delta \mathrm{r} \sim 0,5)$, podendo-se levantar a altura de barreira. Os poços indicam pontos de mínima energia e podem ser caracterizados como reagentes e produtos do processo reativo.

Para avaliar mais de uma variável dinamicamente, tomamos o exemplo da abertura de um anel inorgânico específico, onde observações experimentais identificavam a formação de um anel de cinco membros ${ }^{27}$.

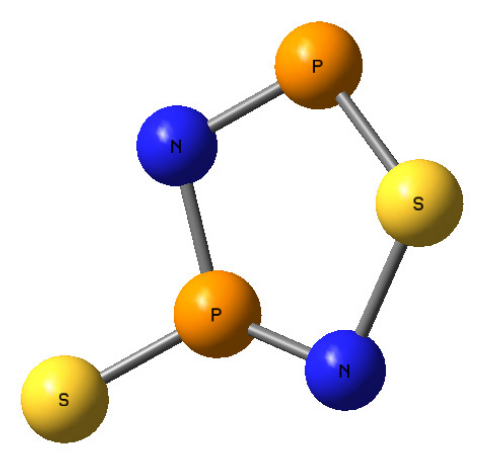

Produto

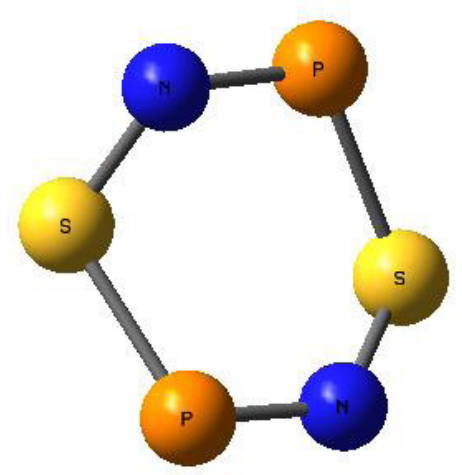

Reagente

Figura 5: Reagente e produto do processo de isomerização de um anel inorgânico de seis membros em um anel de cinco membros. 
Este processo é regido mediante a quebra da ligação N-S, que por consequência potencializava a formação da ligação N-P. Esse processo reativo pode ser representado através do levantamento energético dos parâmetros geométricos sob análise. O maior poço energético representa o produto e os poços intermediários representam os intermediários que levam a formação do produto.

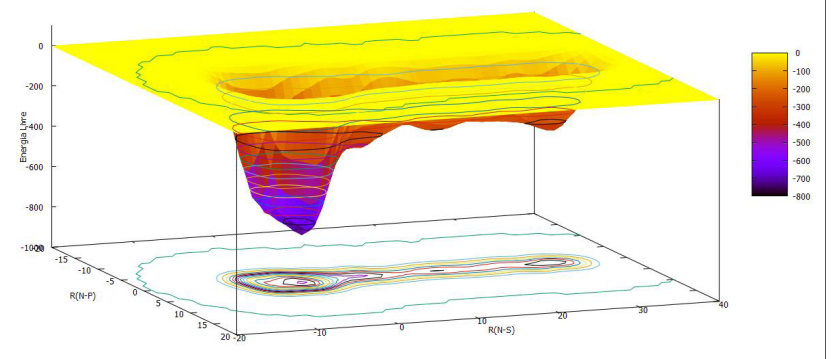

Figura 6: Superfície de energia livre considerando as coordenadas reativas: distâncias R(N-P) e R(N-S).

\section{Conclusões}

Esta comunicação apresentou de forma resumida um guia básico sobre algumas das principais dinâmicas moleculares, sendo que, especificamente, o guia é válido apenas para o CPMD, como já mencionado previamente.

Espera-se que o leitor tenha compreendido, mesmo que de maneira generalizada, a construção de um input, os comandos de cálculo e algumas possíveis análises provenientes dos cálculos de dinâmica molecular.

É acompanhado o crescimento exponencial do uso de dinâmica molecular nos dias atuais em pesquisas e é importante ter uma visão sobre o assunto e suas aplicabilidades.

A presente comunicação, sendo assim, será uma ferramenta auxiliar para os leitores, bem como uma breve fonte de informações. É incentivado o aprofundamento do leitor por meio dos artigos citados neste.

\section{Referências Bibliográficas}

1. Borges, A. W. F. Estudo da estrutura eletrônica do RuCl2 e RuCl3 em solução aquosa utilizando dinâmica molecular de Car-Parrinello. 81 (2011).

2. COUTINHO, N. . Sobre a Origem da Energia de Ativação Negativa na Taxa de Reação do OH + HBr: Estudo do Comportamento NãoArrhenius Usando Dinâmica Molecular de Born-Oppenheimer. (2014).

3. Mueller, M. R. \& Truhlar, D. G. Fundamentals of Quantum Chemistry: Molecular Spectroscopy and Modern Electronic Structure Computations. Physics Today 55, (2002).

4. Namba, A. M., Da Silva, V. B. \& Da Silva, C. H. T. P. Dinâmica molecular: Teoria e aplicações em planejamento de fármacos. Eclet. Quim. 33, 13-24 (2008).

5. Toledo, E. M. ESTUDO DAS PROPRIEDADES ESTRUTURAIS DA CURCUMINA NO VÁCUO USANDO DINÂMICA MOLECULAR DE CAR-PARRINELLO. Programa Pós-graduação Strict. Sensu em Ciências Mol. 1, 94 (2013).

6. Stillinger, F. H. \& Rahman, A. Improved simulation of liquid water by molecular dynamics. J. Chem. Phys. 60, 1545-1557 (1974).

7. Ehrenfest, P. Bemerkung über die angenäherte Gültigkeit der klassischen Mechanik innerhalb der Quantenmechanik. Zeitschrift für Phys. 45, 455-457 (1927).

8. Marx, D. \& Hutter, J. Ab initio molecular dynamics: Theory and implementation. Modern methods and algorithms of quantum ... 1, (2000).

9. Born, M. \& Oppenheimer, R. Zur Quantentheorie der Molekeln. Ann. Phys. 389, 457-484 (1927).

10. Car, R. \& Parrinello, M. Unified Approach for Molecular Dynamics and Density-Functional Theory. Phys. Rev. Let. 55, 2471-2474 (1985).

11. Martiniano, H. F. M. C. Dinâmica Molecular Ab Initio: Aplicações ao Estudo de Propriedades Electrónicas de Sistemas Moleculares. (2012). doi:10.1017/CBO9781107415324.004

12. Yang, P. Car-Parrinello Molecular Dynamics : Final Project. 1-20 (2015).

13. Kohn, W. \& Sham, L. J. Self-Consistent Equations Including Exchange and Correlation Effects. Phys. Rev. 140, A1133-A1138 (1965).

14. Li, X., Tully, J. C., Schlegel, H. B. \& Frisch, M. J. Ab initio Ehrenfest dynamics. J. Chem. Phys. 123, 1-7 (2005).

15. Kumar, P. P., Kalinichev, A. G. \& Kirkpatrick, R. J. Dissociation of carbonic acid: Gas phase energetics and mechanism from ab initio metadynamics simulations. J. Chem. Phys. 126, (2007). 


\section{Artigo Convidado 1}

16. Laio, A. \& Gervasio, F. L. Metadynamics: a method to simulate rare events and reconstruct the free energy in biophysics, chemistry and material science. Reports Prog. Phys. 71, 126601 (2008).

17. Barducci, A., Bonomi, M. \& Parrinello, M. Metadynamics. Wiley Interdiscip. Rev. Comput. Mol. Sci. 1, 826-843 (2011).

18. Roeppstorff, G. Path Integral Approach to Quantum Physics. (Springer Verlag, 1996).

19. Tuckerman, M. E. \& Martyna, G. J. Understanding Modern Molecular Dynamics: Techniques and Applications. J. Phys. Chem. B 104, 159-178 (2000).

20. Madeira, L. \& Vitiello, S. A função radial de distribuição de pares para sistemas Lennard-Jones bidimensionais. Rev. Bras. Ensino Física 34, 7 (2012).

21. SANTIN, L. G. Estudo Teórico das Propriedades Geométricas da Curcumina em Metanol Usando Dinâmica Molecular de CarParrinello. Programa Pós-graduação Strict. Sensu em Ciências Mol. 1, 105 (2014).

22. Agmon, N. The residence time equation. Chem. Phys. Lett. 497, 184-186 (2010).

23. CPMD. Car-Parrinello Molecular Dynamics Manual: An ab initio Electronic Structure and Molecular Dynamics Program. 202 (2015).

24. Martínez, L., Andrade, R., Birgin, E. G. \& Martínez, J. M. Packmol: A package for building initial configurations for molecular dynamics simulations. J. Comput. Chem. 30, 2157-2164 (2009).

25. Martínez, J. M. \& Martínez, L. Packing optimization for automated generation of complex system's initial configurations for molecular dynamics and docking. J. Comput. Chem. 24, 819-825 (2003).

26. Humphrey, W., Dalke, A. \& Schulten, K. VMD: visual molecular dynamics. J. Mol. Graph. 14, 33-8, 27-8 (1996).

27. Zeng, X. et al. SN2P2: A neutral five-membered sulfur-pnictogen(III) ring. Angew. Chemie - Int. Ed. 54, 1327-1330 (2015).

\section{Flavio O. S. Neto, Hugo G. Machado, Eduardo C. Vaz, Yago S. Silva e Valter $\mathrm{H}$. Carvalho-Silva*}

Grupo de Química Teórica e Estrutural de Anápolis, Campus de Ciências Exatas e Tecnológicas, Universidade Estadual de Goiás, Caixa Postal 459, 75001-970, Anápolis, Goiás, Brasil

*E-mail: fatioleg@gmail.com 